Supplementary Material (ESI) for Chemical Communications

This journal is (c) The Royal Society of Chemistry 2012

\title{
Reactive intermediates-induced potential responses of a polymeric membrane electrode for ultrasensitive potentiometric biosensing
}

\author{
Xuewei Wang ${ }^{a b}$ and Wei Qin* ${ }^{a}$ \\ ${ }^{a}$ Key Laboratory of Coastal Zone Environmental Processes, Yantai Institute of Coastal Zone Research (YIC), \\ Chinese Academy of Sciences (CAS); Shandong Provincial Key Laboratory of Coastal Zone Environmental \\ Processes, YICCAS, Yantai,264003, P. R. China \\ ${ }^{b}$ Graduate University of the Chinese Academy of Sciences, Beijing 100049, P. R. China
}

E-mail:wqin@yic.ac.cn

\section{Experimental section}

Chemicals and reagents

Dinonylnaphthalene sulfonic acid (DNNS) as a $50 \%$ solution in heptane, sodium tetrakis[3,5-bis(trifluoromethyl)phenyl]borate, potassium tetrakis(4-chlorophenyl)borate, 2-nitrophenyl octylether (o-NPOE), high molecular weight poly(vinyl chloride) (PVC), horseradish peroxidase (HRP) (Type VI, essentially salt-free, lyophilized powder, $256 \mathrm{U} / \mathrm{mg}$ solid), glucose oxidase (Type X-S, lyophilized powder, $153 \mathrm{U} / \mathrm{mg}$ solid), D-(+)-glucose, $N, N^{\prime}, N, N$ '-tetramethylbenzidine were purchased from Sigma-Aldrich. Tetrahydrofuran, ethanol, dimethyl sulfoxide (solvent for $N, N^{\prime}, N, N^{\prime}$-tetramethylbenzidine), sodium dihydrogen phosphate, disodium hydrogen phosphate, hydrogen peroxide (the concentration of $\mathrm{H}_{2} \mathrm{O}_{2}$ stock solution was standardized by UV absorption measurement using the absorption coefficient of $43.6 \mathrm{M}^{-1} \mathrm{~cm}^{-1}$ at $240 \mathrm{~nm}^{1}$ ), LiOAc were purchased from Sinopharm Group Co. Ltd. All chemicals were of Selectophore or analytical reagent grade.

ISEs preparation and EMF measurements

Polymeric membranes ( $c a .100 \mu \mathrm{m}$ in thickness) containing $49.5 \mathrm{wt} \% \mathrm{PVC}, 49.5 \mathrm{wt} \%$ NPOE and $1.0 \mathrm{wt} \%$ DNNS were prepared by solvent-casting technique with tetrahydrofuran as the 
casting solvent. Disks of 5-mm diameter were punched from the parent membrane and glued to plasticized PVC tubes (i.d. $3 \mathrm{~mm}$, o.d. $5 \mathrm{~mm}$ ) to fabricate the ion selective electrodes. All electrodes were conditioned overnight in $15 \mathrm{mM} \mathrm{NaCl}$ and the same solution was used as the inner filling medium. All measurements were conducted at room temperature in $50 \mathrm{mM}$ phosphate buffer solution (PBS) at $\mathrm{pH} 7.4$ with magnetic stirring (the sodium ion concentration in this buffer solution was calculated to be $89 \mathrm{mM}$ ).

Electromotive force (EMF) was measured using a CHI 760D electrochemical workstation (Shanghai Chenhua Apparatus Corporation, China) in the galvanic cell: $\mathrm{Ag}, \mathrm{AgCl} / 3 \mathrm{M} \mathrm{KCl} / 1 \mathrm{M}$ LiOAc/sample solution/sensing membrane/15 mM NaCl/Ag, AgCl. A Faraday cage was used to lower the noise.

\section{Glucose measurement in blood samples}

After centrifuging the blood samples at $10000 \mathrm{rpm}$ for $30 \mathrm{~min}^{2}, 10 \mu \mathrm{L}$ of the supernatant was diluted into $1 \mathrm{~mL}$ using $50 \mathrm{mM}$ PBS (pH 7.4). Then $20 \mu \mathrm{L}$ of the diluted sample solution was mixed with $2 \mathrm{~mL}$ of $50 \mathrm{mM}$ PBS (pH 7.4) containing $0.5 \mathrm{mg} / \mathrm{mL}$ glucose oxidase. After $4 \mathrm{~min}$, $0.02 \mathrm{mM} \mathrm{N}_{4}$-TMB and $0.1 \mathrm{U} / \mathrm{mL}$ HRP were added to produce the intermediates. The potential increases in the first ten seconds after initiating $N_{4}$-TMB oxidation were used for quantification. For comparison, the glucose concentrations in the same blood samples were determined without dilution using a konbes ${ }^{\mathrm{TM}}$ CDC-2 blood glucose meter (Cambridge Health Care Ltd., Nanjing, China). 


\section{Effect of ion exchanger on the potential responses toward intermediates}

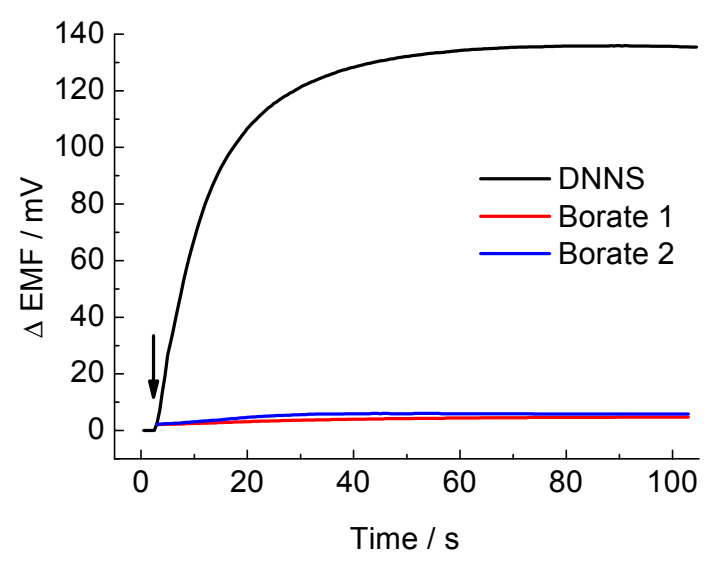

Fig. S1 Potential responses of polymeric membrane electrodes doped with different ion exchangers toward the oxidation of $N_{4}$-TMB $\left(0.02 \mathrm{mM} N_{4}\right.$-TMB, $\left.1 \mu_{\mathrm{M} \mathrm{H}} \mathrm{H}_{2}, 0.1 \mathrm{U} / \mathrm{mL} \mathrm{HRP}\right)$. DNNS was compared with two commonly used lipophilic borate salts (also $1.0 \mathrm{wt} \%$ ), sodium tetrakis[3,5-bis(trifluoromethyl)phenyl]borate $\quad$ (Borate $\quad 1) \quad$ and $\quad$ potassium tetrakis(4-chlorophenyl)borate (Borate 2). All other membrane components (49.5 wt\% PVC and 49.5 $\mathrm{wt} \%$ NPOE), conditioning and measuring conditions are the same for the three kinds of electrodes.

\section{Effect of plasticizer on the potential responses toward intermediates}

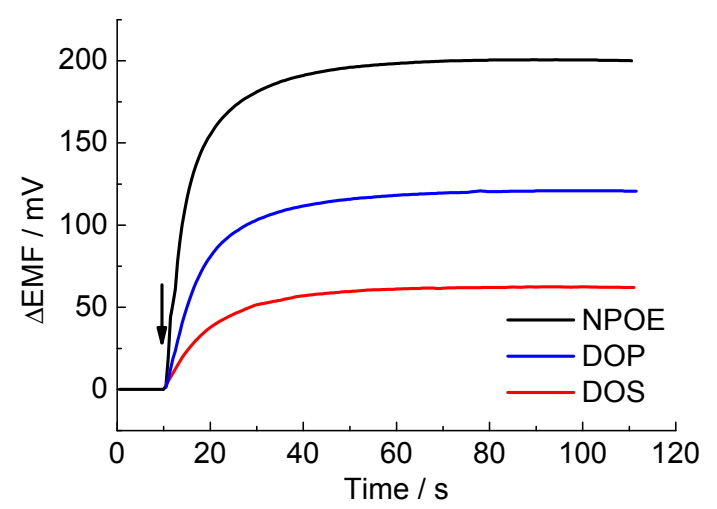

Fig. S2 Potential responses of polymeric membranes with different kinds of plasticizers toward $N_{4}$-TMB oxidation (0.02 mM N4-TMB, $10 \mu \mathrm{M} \mathrm{H} \mathrm{H}_{2} \mathrm{O}_{2}, 0.1 \mathrm{U} / \mathrm{mL}$ HRP). All other membrane components (49.5 wt $\%$ PVC and $1.0 \mathrm{wt} \% \mathrm{DNNS}$ ), conditioning and measuring conditions are the same for the three kinds of electrodes. The larger response of NPOE-based membrane is probably due to the better solvation ability of the polar plasticizer NPOE for intermediates. 


\section{Potential response-reaction time profile}

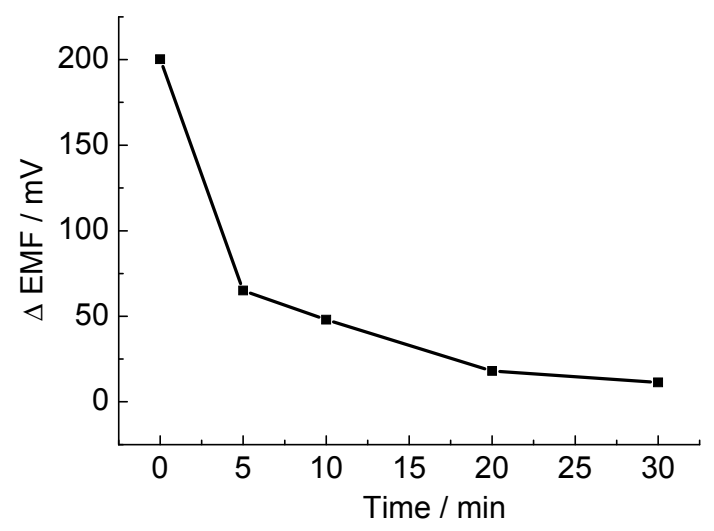

Fig. S3 Potential responses induced by reaction mixtures of $N_{4}$-TMB oxidation at different time points (the reaction condition is identical with that in Fig. 1). The potential change at 0 min represents the real-time response, while those at other time points are obtained by moving the electrode from background solution (PBS) into the corresponding reaction mixtures. All data were plotted using the largest potential increases in the initial $100 \mathrm{~s}$.

\section{Glucose determination in blood samples}

Tab. S1 Results of blood glucose determination using the proposed intermediate-sensitive electrode and commercial glucose meter

\begin{tabular}{ccc}
\hline Samples & $\begin{array}{c}\text { Proposed method }^{\mathrm{a}} \\
\mu \mathrm{M}\end{array}$ & Glucose meter $^{\mathrm{b}}$ \\
& $0.39 \pm 0.04$ & $\mathrm{mM}$ \\
\hline Serum 1 & $0.55 \pm 0.06$ & $4.3 \pm 0.2$ \\
Serum 2 & $0.44 \pm 0.03$ & $5.2 \pm 0.4$ \\
Serum 3 & $5.3 \pm 0.4$ \\
\hline
\end{tabular}

${ }^{\mathrm{a}}$ : Blood glucose concentrations were determined after a 10,000-fold dilution. Data represent an average \pm standard deviation for three measurements.

${ }^{\mathrm{b}}$ : Blood glucose concentrations were determined without dilution. Data represent an average \pm standard deviation for three measurements. 


\section{Regenerability and stability of the intermediate-sensitive electrode}

To reuse the electrode, a 20 min-washing step using a mixture of ethanol and $2 \mathrm{M} \mathrm{NaCl}(1 / 4$, $\mathrm{v} / \mathrm{v})$ was employed. With the regeneration step, the intermediate-sensitive electrode could be repeatedly used for at least five cycles (Fig. S4). No obvious change in potential responses of the intermediate-sensitive electrode was observed after being stored at $4{ }^{\circ} \mathrm{C}$ in the conditioning solution for two weeks.

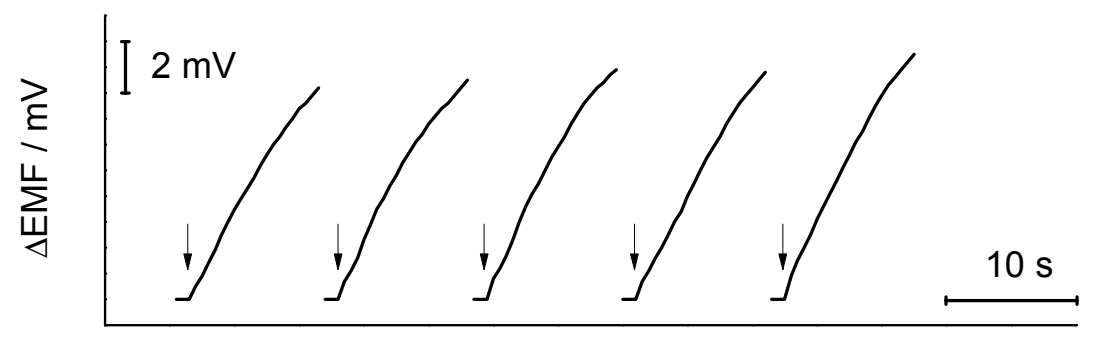

Time

Fig. S4 Potential responses of one intermediate-sensitive electrode toward $N_{4}$-TMB oxidation $(0.02$ $\mathrm{mM} \mathrm{N} \mathrm{N}_{4}$-TMB, $50 \mathrm{nM} \mathrm{H} \mathrm{H}_{2} \mathrm{O}_{2}, 0.1 \mathrm{U} / \mathrm{mL} \mathrm{HRP}$ ) in five consecutive measurements. Arrows indicate the initiation of oxidation reactions.

\section{References}

1. R. F. Beers and I.W. Sizer, J. Biol. Chem., 1952, 195, 133.

2. W. Shi, X. Zhang, S. He and Y. Huang, Chem. Commun., 2011, 47, 10785 